\title{
EFETIVIDADE DOS PROGRAMAS DE APRENDIZAGEM SOCIOEMOCIONAL: O IMPACTO DA SATISFAÇÃO DOS PARTICIPANTES SOBRE OS RESULTADOS
}

\author{
Vanda Sousa \\ Académico Torres Vedras \\ Centro de Investigação em Psicologia para o Desenvolvimento \\ vanda.ps.sousa@gmail.com \\ Vítor Alexandre Coelho \\ Académico Torres Vedras; \\ Centro de Investigação em Psicologia para o Desenvolvimento
}

Recepción Artículo: 27 octubre 2021 Admisión Evaluación: 27 octubre 2021 Informe Evaluador 1: 28 octubre 2021 Informe Evaluador 2: 29 octubre 2021 Aprobación Publicación: 30 octubre 2021

\section{RESUMO}

A Aprendizagem SocioEmocional (ASE) tem ganho reconhecimento, quer no meio científico, quer no campo da prática, com uma crescente demanda para que sejam estudados, não só os seus resultados, mas também a efetividade diferencial destes programas e os factores que influenciam os seus resultados. Os níveis de envolvimento dos participantes nestes programas tem sido apontado como uma variável influente nos seus resultados, no entanto, poucos são os estudos que testam o papel desta variável. No presente estudo são analisados os resultados diferenciais de programas ASE em função dos níveis de envolvimentos dos participantes, considerando a satisfação dos participantes com o programa. Fizeram parte deste estudo 632 participantes, do $4^{\circ}$ ao $8^{\circ}$ ano de escolaridade, que participaram num programa ASE ( $M_{\text {idade }}=10.89$; $D P=1.45 ; 49.8 \%$ raparigas). Foram avaliados os níveis iniciais e finais de competências socioemocionais (Autocontrolo, Consciência Social, Competências Relacionais, Tomada de Decisão), assim como os níveis de satisfação com o programa, tendo sido criados três grupos quanto ao grau de satisfação. Para análise dos dados foram utilizadas ANOVAs de medidas repetidas. Não se observou, para nenhuma das dimensões consideradas, efeitos de interação significativos entre tempo e grau de satisfação, indicando estes resultados que o grau de satisfação não tem um impacto significativo na eficácia dos programas. São identificadas limitações do estudo e discutidas implicações práticas, salientando estes resultados que a satisfação não avalia a eficácia dos programas, sendo um erro operacionalizar o sucesso destes com base na satisfação.

Palavras-chave: programa de aprendizagem socioemocional; satisfação; eficácia 


\title{
EFETIVIDADE DOS PROGRAMAS DE APRENDIZAGEM SOCIOEMOCIONAL: O IMPACTO DA SATISFAÇÃO DOS PARTICIPANTES SOBRE OS RESULTADOS
}

\begin{abstract}
Social and Emotional Learning Programs effectivity: The impact of participants' satisfaction upon results. Social and Emotional Learning (SEL) has been gaining recognition in practice and research, and there is an increasing demand to assess not only SEL program results, but also the differential effectiveness and factors that influence the programs results. The levels of engagement of the participants in this kind of programs are often mentioned as an influential variable in the results. However, little evidence is found about its role. In this study we analyzed the differential results of a SEL program considering the engagement levels, namely the satisfaction of the participants with the program. This study had 632 participants, from $4^{\text {th }}$ to $8^{\text {th }}$ grade that took part in a SEL program $\left(M_{a g e}=10.89 ; S D=1.45 ; 49.8 \%\right.$ girls $)$. Initial and final levels of social and emotional competence were assessed (Self-Control, Social Awareness, Relationship Skills, Decision Making) as well as the level of participants' satisfaction with the program. Three groups were composed regarding the level of satisfaction. To analyze the data, repeated measures ANOVAs were used. There were no significant results for the interaction effect between time and level of satisfaction, for any of the competences, indicating that the satisfaction does not have an impact upon programs results. Limitations and practical implications of this study are discuss, highlighting this results the misconception that is using a measure of satisfaction to asses results of social and emotional learning programs.
\end{abstract}

Keywords: social and emotional learning program; satisfaction; efficacy

\section{INTRODUÇÃo}

As competências socioemocionais são consideradas como essenciais para o sucesso pessoal e escolar, com a literatura a evidenciar o impacto positivo dos programas de aprendizagem socioemocional, quer ao nível do sucesso académico (Durlak et al., 2011), quer ao nível dos comportamentos disruptivos e da violência entre pares (Taylor et al., 2017; Weissberg et al., 2015), tendo-se observado uma crescente demanda para que sejam integrados em meio escolar programas universais de aprendizagem socioemocional (Domitrovich et al., 2017). Assim, tem-se simultaneamente assistido a um crescimento no financiamento para a implementação destes programas (McKown, 2019) e à proliferação destas intervenções (Taylor et al., 2017). Todo este desenvolvimento levou a um crescente apelo na avaliação destes programas, não só no sentido de aferir os seus resultados, isto é, se têm um impacto positivo nas competências socioemocionais (Coelho e Sousa, 2018; Domitrovich et al. 2017) e a extensão do seu impacto, como por exemplo sobre o desempenho académico (Cristóvão et al., 2017). Adicionalmente, vários autores têm defendido que as experiências e pontos de vistas das crianças devem ser tidas em consideração na conceção e implementação de programas de promoção da saúde mental em meio escolar (Mertens, 2014; Cook-Sather, 2019), sendo este elemento da implementação das intervenções considerado como "justo, razoável e apropriado" (Villarreal et al., 2015).

Desta forma, podemos considerar que a investigação se tem focado progressivamente em como conduzir uma avaliação que seja útil para as comunidades em que são desenvolvidos (McKown, 2019), bem como em compreender quais os factores relevantes nestes resultados, i.e., a efetividade diferencial (Coelho e Sousa, 2018).

\section{A Aprendizagem Socioemocional}

A Aprendizagem SocioEmocional (ASE) é definida pelo Collaborative for Academic, Social, and Emotional Learning (CASEL, 2012, p.4) como "o processo pelo qual as crianças e os adultos adquirem e aplicam com eficácia o conhecimento, atitudes e competências necessárias para compreender e gerir emoções, estabelecer e atingir objetivos, sentir e mostrar empatia, estabelecer e manter relações interpessoais positivas e proceder a uma tomada de decisão responsável". A CASEL (2015) identifica cinco áreas interrelacionadas de competências cognitivas, sociais e emocionais: a) a Autoconsciência, englobando a habilidade para reconhecer emoções e pensamentos do próprio e dos outros e compreender a sua influência no comportamento; b) a Consciência social, referindo-se à habilidade de tomar perspetiva e desenvolver empatia com os outros, particularmente de culturas diferentes, e de compreender normas sociais e éticas de comportamento; c) o Autocontrolo, como a habilidade para 
regular as emoções, pensamentos e comportamento, incluindo o controlo de impulsos; d) a Tomada de decisão responsável, consistindo na habilidade de realizar escolhas construtivas relativas ao próprio e em contexto de interação social, integrando nestes processos princípios éticos, normas sociais e a avaliação de consequências; e) as Competências relacionais, referindo-se à habilidade para estabelecer e manter relações saudáveis com diversos indivíduos e grupos, o que inclui uma comunicação clara, a escuta ativa, a cooperação, resistência à pressão de pares, a gestão de conflitos.

Posteriormente, uma meta-análise realizado por Durlak et al. (2011) identificou um conjunto de características que levavam a que os programas ASE apresentassem resultados mais positivos, nomeadamente, a importância dos programas serem sequenciais, trabalhando-se articuladamente, a partir de componentes mais simples para as mais complexas; a utilização de metodologias ativas de aprendizagem; serem focados, alocando-se um tempo definido e suficiente para o desenvolvimento destas atividades; e explícitos, em que os participantes estão cientes dos objetivos, que deverão ser específicos e claramente definidos.

\section{A Avaliação de Programas de Aprendizagem Socioemocional}

Com a proliferação destes programas, ao longo dos anos tem estado também presente a preocupação com a sua avaliação e eficácia (Durlak et al. 2011; Domitrovich et al., 2017; McKown, 2019), nomeadamente pela importância de se poderem determinar quais as intervenções que estão a ser continuamente eficazes e com que populações. Outra das preocupações refere-se ao estudo de variáveis moderadoras, no sentido de se compreender como é que se podem otimizar estas intervenções (Domitrovich et al., 2017; Coelho e Sousa, 2018). Estudos recentes conduzidos em Portugal realçaram algumas destas variáveis que podem servir para promover ou reduzir o impacto dos programas ASE, como 0 ano de escolaridade, meio da escola ou mesmo 0 contexto de aplicação do programa. Coelho, Marchante e Sousa (2016) concluíram que a aplicação de programas similares em diferentes ciclos de estudos levava a resultados mais positivos quando era aplicado no ciclo de estudos mais baixo. Coelho e Sousa (2017) reportaram que um formato de programa levava a resultados mais eficazes do que o formato de currículo para os alunos de $2^{0}$ ciclo. Também Coelho e Sousa (2018) concluíram que 0 contexto em que os programas decorrem tem impacto nos resultados, com os programas inseridos no currículo escolar a obterem resultados mais positivos que os que decorriam em horário não-escolar.

No entanto, no contexto nacional destaca-se a avaliação da perceção que os participantes têm sobre as próprias intervenções, apesar desta ter menos enfoque na literatura internacional. Os únicos manuais elaborados pelo Estado Português (pelo Ministério da Educação e pela Direção Geral da Saúde) na área do desenvolvimento de competências socioemocionais preveem direta (Matos, 1997) ou indiretamente (Direção Geral da Saúde, 2016) apenas a avaliação da satisfação e não a avaliação de resultados. A lógica inerente é a de que os modelos de avaliação deste tipo de intervenções devem incluir o envolvimento/responsividade dos participantes nos programas, nomeadamente com base na premissa de que esta pode influenciar os resultados das intervenções (Arora et. al, 2016). Esta questão é bastante referida no campo da educação, Calderon (2013) deixa saliente que a perceção sobre a aprendizagem é um constructo distinto da aprendizagem em si, refletindo a satisfação com a aprendizagem e não os seus resultados e que a satisfação com a aprendizagem é um fator relevante que deve ser tido em conta no desenvolvimento de programas de aprendizagem. Outros autores salientam ainda a importância de envolver os estudantes, tendo em consideração as suas perceções sobre os programas, nomeadamente para se aferir a adequação destes, favorecendo o seu desenvolvimento e implementação (Mertens, 2014; Cook Sather et al., 2019). No campo da aprendizagem socioemocional e apesar dos apelos, a nível internacional não se encontraram estudos que tivessem em consideração a satisfação dos participantes enquanto moderador dos resultados da intervenção.

\section{Projeto Atitude Positiva}

0 projeto Atitude Positiva surgiu em 2004, estando presentemente a ser implementado nos municípios de Torres Vedras e Cadaval, distrito de Lisboa (Portugal) incluindo entre as suas principais ações, três programas 


\section{EFETIVIDADE DOS PROGRAMAS DE APRENDIZAGEM SOCIOEMOCIONAL: O IMPACTO DA SATISFAÇÃO DOS PARTICIPANTES SOBRE OS RESULTADOS}

de aprendizagem socioemocional ajustados à fase desenvolvimentista: um programa para $04^{0}$ ano (descrito em Coelho, Sousa e Figueira, 2016), um para alunos de $2^{0}$ ciclo (5ํㅡ e $6^{0}$ anos; Coelho e Sousa, 2016) e um para 0

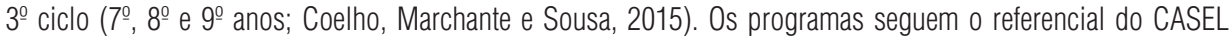
(2012, 2015), apresentando como objetivos promover a autoconsciência, a consciência social, o autocontrolo, as competências relacionais e a tomada de decisão responsável e adotando as indicações metodológicas referidas por Durlack et al. (2011). Os programas são universais e são inseridos no currículo escolar, sendo implementados nos grupos naturais de turma, por psicólogos, de acordo com o respetivo manual.

\section{OBJETIVOS DA INVESTIGAÇÃO}

0 objetivo deste estudo é o de analisar se a satisfação dos participantes de programas de aprendizagem socioemocional vai influenciar a efetividade destes programas. Desta forma, será primeiro necessário analisar se o programa está associado a resultados positivos nas competências socioemocionais avaliadas (autocontrolo, consciência social, competências relacionais e tomada de decisão responsável), tal como proposto em Coelho, Marchante e Sousa (2016), o que leva à formulação das primeiras quatro hipóteses: Os alunos que participaram num programa ASE apresentam uma evolução positiva no Autocontrolo (Hipótese um); na Consciência Social (Hipótese dois); nas Competências Relacionais (Hipótese três) e na Tomada de Decisão Responsável (Hipótese quatro). Também decorrente de resultados anteriores (Coelho, Marchante e Sousa, 2016) será importante verificar se os resultados da eficácia dos programas ASE são mais positivos no ciclo de estudos mais baixo (Hipótese cinco).

Relativamente à questão central do estudo, e dada a inexistência de estudos que avaliem a satisfação dos participantes enquanto variável moderadora da efetividade de programas ASE, optámos pela formulação da seguinte questão de investigação: Será que grupos com diferentes níveis de satisfação com a participação num programa ASE apresentam diferenças nos resultados desse programa para as dimensões do Autocontrolo, Consciência Social, Competências Relacionais e Tomada de Decisão Responsável?

\section{MÉTODO}

\section{Participantes}

0 presente estudo inclui 632 participantes do $4^{0}$ ao $8^{0}$ ano ( $M_{\text {idade }}=10.89 ; D P=1.45 ; 49.8 \%$ raparigas), agrupados por ciclo de escolaridade (Tabela 1), que participaram num programa de aprendizagem socioemocional, no ano letivo de 2019/2020, em seis agrupamentos de escolas do distrito de Lisboa.

Relativamente ao atrito, observou-se uma taxa de atrito superior ao usual (8.7\%), o que pode ser essencialmente explicado por dois fatores: a) a um maior fluxo de transferências de escola por parte dos alunos, havendo alunos que não participaram desde 0 início e outros que não participaram até ao final; b) a entrada em confinamento devido à pandemia COVID 19 que implicou que não fosse possível recolher os dados de avaliação final, junto dos alunos que não terminaram na sessão prevista ou que não estiveram presentes nesta. Desta forma, e dado a estratégia analítica empregue, a maioria das análises inclui apenas 577 participantes que apresentavam as duas avaliações completas.

\section{Instrumentos}

Competências SocioEmocionais. Foram utilizadas quatro escalas do Questionário de Competências SocioEmocionais (Coelho, Sousa \& Marchante, 2015; Coelho \& Sousa, 2020), num total de 25 itens. As escalas utilizadas foram: Autocontrolo (ex:: "Espero pela minha vez sem ficar nervoso(a)"; $\alpha=.74$ ); Consciência Social (ex.: "Ajudo o(a)s outro(a)s quanto têm problemas"; $\alpha=.86$ ); Competências Relacionais (ex.: "Quando há problemas escolhem-me como árbitro(a) ou juíz(a)"; $\alpha=.77$ ); Tomada de Decisão Responsável (ex.: "Considero várias alternativas antes de tomar uma decisão"; $\alpha=.78$ ). Todas as escolas são compostas por sete itens, com a exceção da escala de Tomada de Decisão Responsável que é composta por quatro itens. Os itens são respondidos numa escala de quatro pontos (A - Nunca; B - Algumas vezes; C - Frequentemente; D - Sempre). 
Satisfação. A satisfação foi medida através de dois itens da Escala de Satisfação Atitude Positiva (Coelho et al., 2006), relativos à satisfação com as sessões e com as atividades). Os itens são respondidos numa escala de cinco pontos (1 - Muito más; 2 - Más; 3 - Nem boas nem más; 4 - Boas; 5 - Muito Boas). Foi utilizada a média entre os dois itens $(r=0.59, p<0.001, M=4.3, D P=0.70)$.

\section{Procedimento}

Este estudo englobou os estudantes que participaram num dos programas ASE integrado nas atividades escolares, seguindo o Código de Ética e Deontologia da Ordem dos Psicólogos Portugueses, a legislação nacional nesta matéria e contando com a aprovação dos Agrupamentos de Escolas e Encarregados de Educação. Os programas enquadram-se no projeto Atitude Positiva, havendo um programa dirigido para $04^{0}$ ano ( 13 sessões), outro para o segundo ciclo ( 15 sessões) e outro para $03^{0}$ ciclo (13 sessões). As sessões são de uma hora, semanais, decorrendo na primeira sessão atividades de apresentação, na segunda, a recolha de dados relativos à avaliação inicial e na última sessão, a de dados relativos à avaliação final, onde se inclui os da satisfação. Nas restantes sessões são trabalhados vários temas de acordo com o público a que se destinam (Coelho \& Figueira, 2011).

\section{Análise de Dados}

Para a análise de dados foram utilizadas ANOVAs de medidas repetidas, com o "tempo" (avaliação inicial vs. avaliação final) como fator intrassujeitos e "nível de satisfação" (nível de satisfação mais baixo vs. nível de satisfação intermédio vs. nível de satisfação mais alto) como factor intersujeitos. Quanto ao efeito do ciclo de escolaridade nos resultados, foram também utilizadas ANOVAs de medidas repetidas, mas tendo como factor intersujeitos a variável "ciclo" ( $1^{0}$ ciclo, $2^{\circ}$ ciclo, $3^{0}$ ciclo). A medida utilizada para estimar o tamanho do efeito usada foi 0 eta quadrado parcial $\left(\eta_{p}^{2}\right)$ derivado da ANOVA.

\section{RESULTADOS}

Relativamente ao nível de satisfação, os participantes foram distribuídos por três grupos: 0 grupo 1 incluí os participantes com o nível de satisfação mais baixo [1 - 3.5, $n=90$ ]; 0 grupo 2 inclui os participantes com um nível de satisfação intermédio, com uma pontuação [3.5 - 4.5, $n=284$ ]; 0 grupo 3 inclui os participantes com um nível de satisfação mais elevado, com a pontuação máxima (5, n= 203).

Tabela 1 - Distribuição dos participantes por "ciclo" e por "grupo", estatísticas de teste relativas à distribuição

\begin{tabular}{lcccc}
\hline & $\begin{array}{c}\text { Grupo } 1 \\
\mathrm{n}=90(15.6 \%) \\
\text { resíduos ajust. }\end{array}$ & $\begin{array}{c}\text { Grupo 2 } \\
\mathrm{n}=284(49.2 \%) \\
\text { resíduos ajust. }\end{array}$ & $\begin{array}{c}\text { Grupo 3 } \\
\mathrm{n}=203(35.5 \%) \\
\text { resíduos ajust. }\end{array}$ & $\begin{array}{c}\text { Total } \\
\mathrm{n}(\%)\end{array}$ \\
\hline $1^{\circ}$ ciclo (4 ano) & $23(25.6 \%)$ & $79(27.8 \%)$ & $94(46.3 \%)$ & $196(34.0 \%)$ \\
& -1.8 & -3.1 & 4.6 & $187(32.4 \%)$ \\
$2^{\circ}$ ciclo $\left(6^{\circ}\right.$ ano $)$ & $40(44.4 \%)$ & $101(35.6 \%)$ & $46(22,7 \%)$ & -3.7 \\
$3^{\circ}$ ciclo $\left(7^{\circ} / 8^{\circ}\right.$ ano $)$ & 2.7 & 1,6 & $63(31.0 \%)$ & $194(33.6 \%)$ \\
& $27(30 \%)$ & $104(36.6 \%)$ & -1.0 & \\
\hline
\end{tabular}

Observou-se que a distribuição dos participantes por "ciclo" e "nível de satisfação" não é homogénea (Tabela 1), com os resíduos ajustados a indicarem que o grupo do $1^{0}$ ciclo apresentou um número de participantes acima do esperado no "nível de satisfação mais alto" e abaixo nos restantes, acontecendo o inverso com o grupo do $2^{0}$ ciclo, com $03^{0}$ ciclo a apresentar uma distribuição dentro do esperado. 


\section{EFETIVIDADE DOS PROGRAMAS DE APRENDIZAGEM SOCIOEMOCIONAL: O IMPACTO DA SATISFAÇÃO DOS PARTICIPANTES SOBRE OS RESULTADOS}

Inicialmente foram analisados os potenciais efeitos principais. Observou-se um efeito principal significativo quanto ao "tempo" para todas as dimensões: Autocontrolo, $F(1,574)=15.969, p<.001, \eta_{p}{ }^{2}=.027$, Consciência Social, $F(1,574)=8.420, p=.014, \eta_{p}^{2}=.014$, Competências Relacionais $F(1,574)=4.264, p=.039, \eta_{p}^{2}=$ .007, Tomada de Decisão Responsável $F(1,574)=22.105, p<.001, \eta_{p}^{2}=.037$, indicando um aumento significativo nas competências do momento inicial de avaliação para o final.

Observou-se também um efeito principal significativo quanto ao "nível de satisfação" para todas as dimensões: Autocontrolo nas Relações Sociais, $F(2,574)=38.233, p<.001, \eta_{p}{ }^{2}=.118$, Consciência Social, $F(2,574)$ $=64.928, p<.001, \eta_{p}{ }^{2}=.184$, Competências Relacionais $F(2,574)=25.050, p<.001, \eta_{p}{ }^{2}=.080$, Tomada de Decisão Responsável $F(2,574)=46.214, p<.001, \eta_{p}{ }^{2}=.139$, observando-se na Tabela 1 que 0 Grupo 1 apresentou níveis de competências socioemocionais mais baixos, seguindo-se 0 Grupo 2, com 0 Grupo 3 a apresentar níveis de competências mais elevados.

Nas análises relativas ao efeito de interação entre "tempo" e "ciclo", observou-se que não houve resultados significativos para o Autocontrolo, $F(2,574)=0.848, p=.429, \eta_{p}^{2}=.003$, Consciência Social, $F(2,574)=0.024$, $p=.976, \eta_{p}^{2}=.000$, Competências Relacionais $F(2,574)=0.217, p=.805, \eta_{p}^{2}=.001$. Observou-se um efeito significativo para a Tomada de Decisão Responsável, $F(2,574)=11.767, p=.019, \eta_{p}^{2}=.014$, com $01^{0}$ ciclo a apresentar um acréscimo mais acentuado, $M_{T 1}=7.08, D P_{T 1}=2.56, M_{T 2}=7.96, D P_{T 2}=2.49$, seguido do $3^{0}$ ciclo, $M_{T 1}=6.28, D P_{T 1}=2.07, M_{T 2}=6.72, D P_{T 2}=2.13$, com o 2ํㅡㄴ ciclo com um menor acréscimo, $M_{T 1}=$ 6.66, $\left.D P_{T 1}=2.57, M_{T 2}=6.85, D P_{T 2}=2.53\right)$.

Tabela 2 - Estatísticas descritivas da avaliação inicial e final, por nível de satisfação, com as estatísticas de teste relativas ao efeito de interação "tempo" $x$ "grupo"

\begin{tabular}{|c|c|c|c|c|c|c|c|c|}
\hline & \multicolumn{2}{|c|}{$\begin{array}{c}\text { Grupo } 1 \\
\text { Nível de satisfação } \\
\text { mais baixo } \\
(n=90)\end{array}$} & \multicolumn{2}{|c|}{$\begin{array}{c}\text { Grupo } 2 \\
\text { Nível de satisfação } \\
\text { intermédio } \\
(n=284)\end{array}$} & \multicolumn{2}{|c|}{$\begin{array}{c}\text { Grupo } 3 \\
\text { Nível de satisfação } \\
\text { mais alto } \\
(n=203)\end{array}$} & \multirow[t]{2}{*}{$F$} & \multirow[t]{2}{*}{$p$} \\
\hline & $\begin{array}{c}\text { Inicial } \\
M(D P)\end{array}$ & $\begin{array}{c}\text { Final } \\
M(D P)\end{array}$ & $\begin{array}{c}\text { Inicial } \\
M(D P)\end{array}$ & $\begin{array}{c}\text { Final } \\
M(D P)\end{array}$ & $\begin{array}{c}\text { Inicial } \\
M(D P)\end{array}$ & $\begin{array}{c}\text { Final } \\
M(D P)\end{array}$ & & \\
\hline Autocontrolo & 12.27 & 13.11 & 14.17 & 14.68 & 15.73 & 16.29 & .33 & .72 \\
\hline Consciência Social & 11.20 & 11.38 & 13.64 & 14.20 & 15.99 & 16.94 & 1.18 & .31 \\
\hline Comp. Relacionais & 8.44 & 8.60 & 9.33 & 9.46 & 10.88 & 11.66 & 2.07 & .13 \\
\hline Tomada de Decisão & 5.48 & 5.98 & 6.45 & 6.76 & 7.52 & 8.31 & 2.25 & .11 \\
\hline
\end{tabular}

De acordo com a Tabela 2, não se observou qualquer efeito de interação significativo entre "tempo" e "nível de satisfação". Dado que se observou uma distribuição heterógenea entre "nível de satisfação" e "ciclo", repetiuse a análise, inserindo-se como covariável "ciclo", a qual também não revelou qualquer efeito de interação entre "tempo" e "nível de satisfação" significativo.

\section{DISCUSSÃO}

0 principal objetivo do presente estudo era analisar se existiam diferenças na eficácia de programas ASE em função do nível de satisfação que os participantes apresentavam com a sua participação nesse mesmo programa. No entanto, para que fosse possível esclarecer essa questão de investigação foi necessário verificar se a presente amostra apresentava um padrão de resultados de eficácia positivos do programa ASE sobre as competências socioemocionais.

Efetivamente todas as primeiras quatro hipóteses foram apoiadas pelos resultados do presente estudo. Em todas as competências socioemocionais analisadas (Autocontrolo, Consciência Social, Competências Relacionais e Tomada de Decisão Responsável) observaram-se aumentos significativos entre a avaliação inicial e a avaliação final. Consequentemente, embora não fosse objetivo deste estudo aferir os resultados do programa (dado não existirem grupos de controlo), este padrão de resultados está alinhado com Coelho, Marchante e 
Sousa (2016) que tinham concluído pela existência de um impacto positivo dos programas ASE dos vários ciclos sobre as competências socioemocionais.

Relativamente à Hipótese cinco, podemos concluir que este foi apenas parcialmente suportada pelos resultados do presente estudo, visto apenas na Tomada de Decisão Responsável terem sido encontradas diferenças entre ciclos relativamente à eficácia dos resultados. 0 resultado mais positivo encontrado entre alunos do $1^{0}$ ciclo pode ser potencialmente explicado por questões desenvolvimentais. Durante este período esta competência começa a ser mais solicitada devido às transições ecológicas que 0 aluno tem de lidar.

No que concerne à questão de investigação central, não se observaram quaisquer efeitos de interação entre tempo e nível de satisfação, indicando que não existiram diferença de impacto nos resultados dos programas ASE de acordo com o nível de satisfação dos participantes. Isto, apesar da análise de dados efetuada ter revelado diferenças significativas nas competências socioemocionais em função dos níveis de satisfação, observando-se no grupo com níveis inferiores de satisfação, níveis de competências socioemocionais mais baixas, enquanto no grupo com níveis de satisfação superiores foram reportados níveis de competências socioemocionais mais elevados.

Consequentemente, os resultados deste estudo, indicam que a satisfação dos participantes com os programas não é um fator de relevo na efetividade dos programas em contexto escolar, indo ao encontro das considerações de Calderon (2013). Este autor referia que a perceção sobre a aprendizagem é um constructo distinto da aprendizagem em si. Corroborando esta ideia estão os resultados relativos ao efeito principal do nível de satisfação que indicam que a perceção sobre o programa é dependente do perfil de competências socioemocionais dos participantes. Desta forma, este estudo fornece evidências face a questões metodológicas no campo da avaliação dos programas ASE, salientando que uma medida de satisfação com os programas não é indicativa do seu impacto e demonstrando que será um erro operacionalizar o sucesso destes com base na satisfação.

\section{CONCLUSÃO}

Apesar de ter existido um impacto positivo dos programas ASE Atitude Positiva aplicados nos vários ciclos de estudo, não existiram diferenças nos níveis de eficácia do programa entre os grupos que reportaram níveis diferentes de satisfação com o programa ASE em que participaram.

A inexistência de diferenças na evolução das competências socioemocionais vem sublinhar a relevância de não se confundir avaliação do processo (avaliação da satisfação dos participantes) com a avaliação de resultados (avaliação do impacto dos programas ASE), contrastando com o preconizado em Portugal por vários agentes institucionais (Matos, 2016; DGE, 2018).

No entanto, os níveis iniciais de competências socioemocionais podem ser preditores da satisfação que os participantes acabam por reportar. Os grupos que apresentarem um nível de satisfação mais baixo também apresentavam um nível mais baixo em todas as competências socioemocionais analisadas.

\section{LIMITAÇÕES E DIREÇÕES FUTURAS}

Este estudo demonstrou a importância de se investigar as perceções dos participantes, apelando não só a medidas mais robustas, mas também que considerem outros aspetos, nomeadamente como numa abordagem mais qualitativa. No entanto, existem duas limitações importantes neste estudo que importam realçar.

Uma das limitações que se deverá apontar a este estudo reside na medida de satisfação que, sendo constituída por apenas dois itens, é mais limitada no que está a medir, com uma variabilidade de dados mais reduzida. A outra limitação deriva da impossibilidade de utilizar grupos de controlo para mais adequadamente avaliar a eficácia dos programas ASE. Neste estudo, tal não foi possível dado os grupos de controlo naturalmente não preencherem avaliação de satisfação relativamente à participação no programa.

Estudos futuros deveriam analisar a questão da efetividade diferencial da satisfação dos participantes de um ângulo diferente. No presente estudo, os resultados indicam que os alunos que apresentam níveis mais baixos 


\section{EFETIVIDADE DOS PROGRAMAS DE APRENDIZAGEM SOCIOEMOCIONAL: 0 IMPACTO DA SATISFAÇÃO DOS PARTICIPANTES SOBRE OS RESULTADOS}

de competências socioemocionais iniciais também reportam níveis mais baixos de satisfação com a participação nos programas ASE. Tal resultado pode-se dever a diferentes fatores (por exemplo, maior dificuldade percebida das tarefas), que deveriam ser identificados em estudos futuros.

\section{REFERÊNCIAS BIBLIOGRAFICAS}

Arora, P. G., Connors, E. H., George, M. W., Lyon, A. R., Wolk, C. B., \& Weist, M. D (2016). Advancing EvidenceBased Assessment in School Mental Health: Key Priorities for an Applied Research Agenda. Clinical Child \& Family Psychology Review, 19, 271-284. Doi: 10.1007/s10567-016-0217-y

Calderon, 0. (2013). Direct and Indirect Measures of Learning Outcomes in an MSW Program: What Do We Actually Measure? Journal of Social Work Education, 49, 408-419. D0I: 10.1080/10437797.2013.796767

Coelho, V. A., \& Sousa, V. (2017). Comparing Two Low Middle School Social and Emotional Learning Program Formats: A Multilevel Effectiveness Study. Journal of youth and adolescence, 46(3), 656-667. https://doi.org/10.1007/s10964-016-0472-8

Coelho, V. A., \& Sousa, V. (2018). Differential Effectiveness of a Middle School Social and Emotional Learning Program: Does Setting Matter?. Journal of youth and adolescence, 47(9), 1978-1991. https://doi.org/10.1007/s10964-018-0897-3

Coelho, V. A., \& Sousa, V. (2020). Validação do questionário de avaliação de competências socioemocionais para alunos de $1^{0} \mathrm{e} 2^{\circ}$ ciclo do ensino básico. International Journal of Developmental and Educational Psychology, 2(1), 431-440. https://doi.org/10.17060/ijodaep.2020.n1.v2.1863

Coelho, V., Freitas, M. \& Sousa, V. (2006). Satisfaction Scale Transição Positiva (not published)

Coelho, V., \& Figueira, A. (2011). Project "Positive Attitude": promoting school success through social and emotional abilities development. Design for elementary and middle school students, in Portugal. Interamerican Journal of Psychology, 45(2), 185-192.

Coelho, V. A., Marchante, M., \& Sousa, V. (2015). "Positive Attitude": A multilevel model analysis of the effectiveness of a Social and Emotional Learning Program for Portuguese middle school students. Journal of Adolescence, 43, 29-38. doi: 10.1016/j.adolescence.2015.05.009

Coelho, V. A., Marchante, M., \& Sousa, V. (2016). Positive Attitude Program's impact upon self-concept across childhood and adolescence. Revista de Psicodidáctica, 21(2), 261-282. doi: 10.1387/RevPsicodidact.15129

Coelho, V. A., Sousa, M. \& Figueira, A. P. (2016). The effectiveness of a Portuguese elementary school social and emotional learning program. Journal of Primary Prevention, 37, 433-447. doi: 10.1007/s10935-016-04454

Coelho, V. A., Sousa, V., \& Marchante, M. (2015). Development and validation of the Social and Emotional Competencies Evaluation Questionnaire. Journal of Educational and Developmental Psychology, 5(1), 139147. doi: 10.5539/jedp.v5n1p139

Collaborative for Academic, Social, and Emotional Learning. (2012). 2013 CASEL guide: Effective social and emotional learning programs (Preschool and elementary school edition). Chicago, IL: Authors.

Collaborative for Academic, Social and Emotional Learning. (2015). CASEL Guide: Effective social and emotional learning programs-middle and high school edition. Collaborative for Academic, Social and Emotional Learning. CASEL, 1-45.

Cook-Sather, A., Krishna Prasad, S., Marquis, E. and Ntem, A. (2019), Mobilizing a Culture Shift on Campus: Underrepresented Students as Educational Developers. Teaching and Learning, 2019, 2130. https://doi.org/10.1002/tl.20345

Direção Geral da Saúde (2016). Manual para a Promoção de Competências Socioemocionais em meio escolar. Lisboa, 2016.

Domitrovich, C. E., Durlak, J. A., Staley, K. C., \& Weissberg, R. P. (2017). Social-Emotional Competence: An Essential Factor for Promoting Positive Adjustment and Reducing Risk in School Children. Child Development, 88(2), 408-416. doi: 10.1111/cdev.12739 
Durlak, J. A., Weissberg, R. P., Dymnicki, A. B., Taylor, R. D., \& Schellinger, K. B. (2011). The Impact of Enhancing Students' Social and Emotional Learning: A Meta-Analysis of School-Based Universal Interventions. Child Development, 82(1), 405-432.

https://doi.org/10.1111/j.1467-8624.2010.01564.x

Matos, M. G. (Ed.) (1997). Manual de Utilização: Programa de promoção da competência social. Lisboa: Ministério da Educação

McKown, C. (2019). Challenges and Opportunities in the Applied Assessment of Student Social and Emotional Learning. Educational Psychologist, 54(3), 205-221. https://doi.org/10.1080/00461520.2019.1614446

Taylor, R.D., Oberle, E., Durlak, J.A. and Weissberg, R.P. (2017)., Promoting Positive Youth Development Through School-Based Social and Emotional Learning Interventions: A Meta-Analysis of Follow-Up Effects. Child Development, 88, 1156-1171. https://doi.org/10.1111/cdev.12864

Villarreal, V., Ponce, C., \& Gutierrez, H. (2015). Treatment acceptability of interventions published in six school psychology journals. School Psychology International, 36(3), 322-332. https://doi.org/10.1177/0143034315574153

Weissberg, R.P., Durlak, J.A., Domitrovich, C.E., \& Gullotta, T.P. (2015). Social and emotional learning: Past, present, and future. In J.A. Durlak, C.E. Domitrovich, R.P. Weissberg \& T.P. Gullotta (Eds.), Handbook of social and emotional learning: Research and practice (pp. 3-19). New York, NY: Guilford. 
